\title{
Reduction of spurious velocity in the free-energy-based lattice Boltzmann method for large density ratio
}

\author{
Jia-ming GONG*, Nobuyuki OSHIMA* and Yutaka TABE** \\ ${ }^{*}$ Division of Mechanical and Space Engineering, Hokkaido University \\ N13W8, Kita-ku, Sapporo 060-6868, Japan \\ E-mail: gjmhit@hotmail.com \\ ${ }^{*}$ Division of Energy and Environmental Systems, Hokkaido University \\ N13W8, Kita-ku, Sapporo 060-6868, Japan
}

Received 20 October 2014

\begin{abstract}
The spurious velocity around curved interface, arising from the calculation of the Poisson equation with staggered grids, is reduced in the free-energy-based two-phase flow lattice Boltzmann method (LBM) for large density ratios. It is found that the pressure calculation from the Poisson equation, using the successive over-relaxation method with staggered grids, would introduce anisotropic discretization errors and lead to deviations of its calculated value from the theoretical prediction. Moreover, the anisotropic pressure would induce a large magnitude of spurious velocity, which is the driving force for droplet shape deformation. By blending the velocity components in the discretization equations of the Poission equation from two types of staggered grids that separately make use of the velocity components in the orthogonal and the diagonal directions, the magnitude of the spurious velocity and the droplet deformation are diminshed. It is found that, by appropriate choice of the blending factor, the magnitude of the spurious velocity can be reduced to half of its original value, and the shape deformation and pressure deviation from the theoretical prediction can be minimized.
\end{abstract}

Key words : Spurious velocity, Anisotropic error, Poisson equation, Lattice Boltzmann method, Water droplet, Shape deformation, Free energy, Successive over-relaxation

\section{Introduction}

The lattice Boltzmann method (LBM), based on microscopic models and mesoscopic kinetic equations for fluids, has attracted increased attention in anticipation of effectively simulating multiphase flows (Succi, 2001). Among the existing multiphase LBM models, the free-energy-based LBM model is becoming popular, for its firmer physical base and simpler algorithm (Huang, et al., 2011).

The free-energy-based LBM model was originally proposed by Swift et al. (1995); however, it did not incorporate Galilean invariance for the viscous term in the Navier-Stokes equation. Inamuro et al. (2000) improved Swift's model by applying an asymptotic theory to ensure that the revised model satisfied the Galilean invariance. Furthermore, by using the projection method at the interface region, Inamuro et al. (2004) extended the model to permit simulations of incompressible two-phase flows with large density ratios yielding stable results, even in the cases where the ratio was as high as 1000:1. Moreover, Lee and Lin (2005) and Lee and Fischer (2006), based on the model devised by He et al. (1999), proposed two models that can simulate two-phase flows at a large density ratio using a stable discretization scheme of the lattice Boltzmann equation. Zheng et al. (2006) also proposed a Galilean-invariant free-energy model, based on Swift's model, and claimed that it is able to mimic two-phase flows with large density differences.

The spurious velocity, also referred to as parasitic current, is an artificial velocity with a small amplitude, that exists in the vicinity of an interface in the simulation. This artificial velocity field is a common problem in the two-phase flow simulation techniques of diffuse interface methods, such as the LBM, the volume of fluid (VOF), the 
phase field (PF) and the level set (LS) methods (Ryu and Ko, 2011; Scarbolo, et al., 2012). This artificial velocity field is nonphysical and arises from a slight force imbalance between the discretized forces in the interface region. Its presence not only degrades the simulation accuracy, but also may cause instability problems. Many efforts have been extended to reduce or eliminate the spurious velocity and identify its origins in the LBM models, especially, in the free-energy-based LBM models (Wagner, 2003; Lee and Fischer, 2006; Pooley and Furtado, 2008; Seta and Okui, 2007; Chiappini, et al., 2010; Guo, et al., 2011; Connington and Lee, 2012). All these publications have already provided a deep insight on the causes of the spurious velocity and have introduced several methods to reduce or eliminate it in the the free-energy-based LBM models. However, as pointed out by Connington and Lee (2012) in their review paper, "The research community has not come to a consensus on the cause, and so too has not come to a consensus on the solution". For the present free-energy-based LBM models that exist in the literature, and in spite of the many common origins of the spurious velocity most of these models share, some proposed models are associated with new or unique sources, due to the special treatments in each detailed model.

The model proposed by Inamuro et al. (2004) has been proved to be reliable. Yan and $\mathrm{Zu}$ (2006) successfully simulated the dynamics of liquid droplets on uniform and heterogeneous wetting walls. Moriyama and Inamuro (2011) and Tabe et al. $(2009,2012)$ quantitatively simulated the two-phase flow of liquid water and gas in the PEFC. In this model, two distribution functions were adopted: the first one was used to predict the density distribution of the two-phase system based on the idea of the Level set method, by adding or keeping an advection term in the function; while the second one was used to predict the velocity field. The velocity calculated from the LBM scheme is not divergence-free, and the continuity equation is satisfied by the the fractional step method that is based on the Poisson equation. The Poisson equation in this model was originally solved by means of an additional LBM scheme, which was very time-consuming. Instead of this approach, Tabe et al. (2009) solved the Poisson equation using the successive over-relaxation (SOR) method for a faster calculation and greater ease of implementation. These special treatments adopted in both publications by Inamuro et al. (2004) and Tabe et al. (2009), render the model quite different from other free-energy-based LBM models. However, so far, no one has ever considered the unique sources of spurious velocity, nor the spurious velocity related phenomena in this model. Furthermore, the thermodynamic meaning of the pressure in the Poisson equation has not been explained before. And, the difference between the calculated pressure from the Poisson equation and the one predicted by the equation of state (EOS) is unclear (Lee and Lin (2005)).

This paper follows the schemes and parameters adopted in Tabe et al. (2009). Based on the simulation results presented herein, the authors provide explanations for the questions raised earlier and propose a new method that reduces the error arising from the pressure calculation from the Poisson equation. In accordance to the author's recent publications (Gong et al. (2011, 2013)), the phenomena related with the spurious velocity around the curved interface are very complicated and are affected by many factors. Focus in this work is maintained on the pressure term in the Poisson equation. Therefore, all the other factors that may complicate explanations on the topic are excluded, confining the discussions in this paper mainly to the simulation results of a water droplet with a moderate radius.

\section{Nomenclature}

$c: \quad$ Characteristic particle speed, $\mathrm{m} / \mathrm{s}$

$c_{i}: \quad$ Restricted velocities of particle ensembles, $\mathrm{m} / \mathrm{s}$

$f_{i}$ : $\quad$ Particle velocity distribution functions for the calculation of an order parameter

$g_{i}: \quad$ Particle velocity distribution functions for the calculation of a predicted velocity

$L$ : $\quad$ Characteristic length, $\mathrm{m}$

$p: \quad$ Pressure, $\mathrm{Pa}$

Sh: $\quad$ Strouhal number

$t: \quad$ Time scale, $\mathrm{s}$

$u, u^{*}: \quad$ Current velocity and predicted velocity, $\mathrm{m} / \mathrm{s}$

$x, y: \quad x, y$ position coordinates, $\mathrm{m}$

$\Delta x: \quad$ Spacing of the squared lattice, $\mathrm{m}$

Greek letters

$\alpha$ : $\quad$ Blending factor of two types of staggered grids 
$\xi: \quad$ Coordinate perpendicular to the interface, $\mathrm{m}$

$\lambda: \quad$ Free Parameter

$\rho: \quad$ Density, $\mathrm{kg} / \mathrm{m}^{3}$

$\sigma: \quad$ Interface tension, $\mathrm{N} / \mathrm{m}$

$\mu: \quad$ Viscosity, $\mathrm{N} \cdot \mathrm{s} / \mathrm{m}^{2}$

$\tau_{f}, \tau_{g}: \quad$ Dimensionless single relaxation times

$\kappa_{f}, \kappa_{g}$ : Constant parameters determining the interface thickness and strength, respectively

$\phi, \phi_{0}$ : Order parameter and reference order parameter, respectively

\section{Superscript and Subscript}

eq: $\quad$ Equilibrium state

$G: \quad$ Gas

$L: \quad$ Liquid

$\alpha, \beta: \quad$ Cartesian coordinates

\section{Numerical scheme}

\subsection{LBM model for large density ratio}

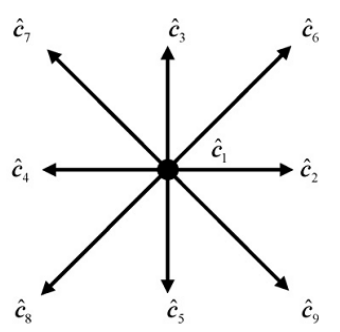

(a) The 2DV9 model

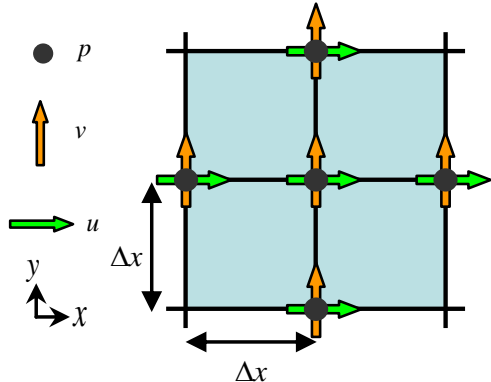

(b) Co-location

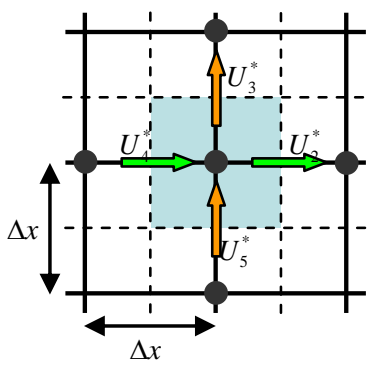

(c) Staggered Grid 1

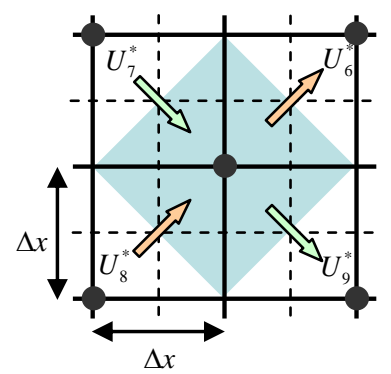

(d) Staggered Grid 2

Fig. 1 The 2DV9 model and lattice configurations used in the LBM and the SOR method.

In the LBM model the two-particle velocity distribution functions, $f_{i}$ and $g_{i}$, are used. The first, $f_{i}$, is used in the calculation of an order parameter $\phi$ that distinguishes two phases; $\phi<\phi_{G}$ corresponds to the gas phase, $\phi_{G} \leq \phi \leq \phi_{L}$ corresponds to the interface, and $\phi>\phi_{L}$ corresponds to the liquid phase. The function $g_{i}$ is used to calculate the predicted velocity of the two-phase fluid, in the absence of a pressure gradient. The evolution functions of the two-particle velocity distribution functions $f_{i}$ and $g_{i}$ are described by Eqs. (1) and (2):

$$
\begin{aligned}
& f_{i}\left(\mathbf{x}+\mathbf{c}_{i} \Delta t, t+\Delta t\right)=f_{i}(\mathbf{x}, t)-\frac{1}{\tau_{f}} \cdot\left(f_{i}(\mathbf{x}, t)-f_{i}^{e q}(\mathbf{x}, t)\right) \\
& g_{i}\left(\mathbf{x}+\mathbf{c}_{i} \Delta t, t+\Delta t\right)=g_{i}(\mathbf{x}, t)-\frac{1}{\tau_{g}}\left(g_{i}(\mathbf{x}, t)-g_{i}^{e q}(\mathbf{x}, t)\right)+\frac{3 E_{i} c_{i \alpha}}{\rho}\left[\frac{\partial}{\partial x_{\beta}}\left\{\mu\left(\frac{\partial u_{\beta}}{\partial x_{\alpha}}+\frac{\partial u_{\alpha}}{\partial x_{\beta}}\right)\right\}\right] \Delta x
\end{aligned}
$$

Here, $f_{i}^{e q}$ and $g_{i}^{e q}$ are the equilibrium distribution functions, $\tau_{f}$ and $\tau_{g}$ are dimensionless single relaxation times, $E_{i}$ are the associated weight coefficients, $\Delta x$ is the spacing of the squared lattice, $\Delta t$ is the time step during which the particles travel across the distance of a lattice spacing, $\rho$ is the density, $\mu$ is the viscosity and $\boldsymbol{u}$ is the current velocity. The subscripts $\alpha$ and $\beta$ represent Cartesian coordinates and the summation convention is used. The last terms on the right side of Eq. (2) represents the effects of viscous stress, and in this paper the gravitation term was not considered. The two-dimensional (2D), 9-velocities model (the 2D9V model) is used in the LBM model, and the lattice velocity directions are shown in Fig. 1(a).

The order parameter, $\phi$, distinguishing the two phases, and the predicted velocity, $\boldsymbol{u}^{*}$, of the multi-phase fluid are defined in terms of the two-particle velocity distribution functions, as shown below:

$\phi=\sum_{i=1}^{9} f_{i}$ 
$\boldsymbol{u}^{*}=\sum_{i=1}^{9} \boldsymbol{c}_{i} g_{i}$

The equilibrium distribution functions $f_{i}^{e q}$ and $g_{i}^{e q}$ in Eqs. (1) and (2) are given by the following equations:

$$
\begin{array}{r}
f_{i}^{e q}=H_{i} \phi+F_{i}\left[p_{0}-\kappa_{f} \phi \frac{\partial^{2} \phi}{\partial x_{\alpha}^{2}}-\frac{\kappa_{f}}{6}\left(\frac{\partial \phi}{\partial x_{\alpha}}\right)^{2}\right]+3 E_{i} \phi c_{i \alpha} u_{\alpha}+E_{i} \kappa_{f} G_{\alpha \beta}(\phi) c_{i \alpha} c_{i \beta} \\
g_{i}^{e q}=E_{i}\left[1+3 c_{i \alpha} u_{\alpha}-\frac{3}{2} u_{\alpha} u_{\alpha}+\frac{9}{2} c_{i \alpha} c_{i \beta} u_{\alpha} u_{\beta}+\frac{3}{2}\left(\tau g-\frac{1}{2}\right) \times \Delta x\left(\frac{\partial u_{\beta}}{\partial x_{\alpha}}+\frac{\partial u_{\alpha}}{\partial x_{\beta}}\right) c_{i \alpha} c_{i \beta}\right] \\
+E_{i} \frac{\kappa_{g}}{\hat{\rho}} G_{\alpha \beta}(\rho) c_{i \alpha} c_{i \beta}-\frac{1}{2} F_{i} \frac{\kappa_{g}}{\rho}|\nabla \rho|^{2}
\end{array}
$$

and $\quad G_{\alpha \beta}(\lambda)=\frac{9}{2} \frac{\partial \lambda}{\partial x_{\alpha}} \frac{\partial \lambda}{\partial x_{\beta}}-\frac{9}{4} \frac{\partial \lambda}{\partial x_{\gamma}} \frac{\partial \lambda}{\partial x_{\gamma}} \delta_{\alpha \beta}$

where $\alpha, \beta, \gamma$ are the Cartesian coordinates following the summation convection, $\delta_{\alpha \beta}$ is the Kronecker delta, $\kappa_{f}$ is a constant parameter that determines the interface width between the two phases and $\kappa_{g}$ is a constant parameter that determines the strength of the interface tension of the liquid water/gas system. Additionally, $\lambda$ is the free parameter used to represent $\phi$ and $\rho$ in Eqs. (5) and (6). Correspondingly, $p_{0}$, is the pressure of the van der Waals fluid described by $\phi$, where the equation of state (EOS) is defined by

$p_{0}=\phi \frac{\partial \psi}{\partial \phi}-\psi=\phi T \frac{1}{1-b \phi}-a \phi^{2}$

Here, $\psi$ is the bulk free-energy density, and $a, b$ and $T$ are free parameters determining the maximum and minimum values of the order parameter $\phi$. In this paper, $a=1, b=6.7, T=3.5 \times 10^{-2}$ were used.

The interface tension is obtained for the liquid water/gas system by

$\sigma=\kappa_{g} \int_{-\infty}^{\infty}\left(\frac{\partial \rho}{\partial \xi_{\rho}}\right)^{2} d \xi_{\rho}$

where $\xi_{\rho}$ are the coordinates perpendicular to the interface of the liquid water/gas.

The densities of the liquid water and gas can be calculated by

$\rho=\left\{\begin{array}{cl}\rho_{G}, & \phi \leq \phi_{G} \\ \frac{\Delta \rho}{2}\left[\sin \left(\frac{\phi-\bar{\phi}}{\Delta \phi} \pi\right)+1\right]+\rho_{G}, & \phi_{G}<\phi<\phi_{L} \\ \rho_{L}, & \phi \geq \phi_{L}\end{array}\right.$

Since the predicted velocity $\boldsymbol{u}^{*}$ calculated by Eq. (4) does not satisfy the continuity equation $\left(\nabla \cdot \boldsymbol{u}^{*}=0\right)$, the correction of $\boldsymbol{u}^{*}$ is needed. The current velocity, $\boldsymbol{u}$, that satisfies the continuity equation can be obtained by the fractional step method with the following equations:

$$
\begin{aligned}
& \nabla \cdot\left(\frac{\nabla p}{\rho}\right)=S h \frac{\nabla \cdot \boldsymbol{u}^{*}}{\Delta t} \\
& \operatorname{Sh} \frac{\boldsymbol{u}-\boldsymbol{u}^{*}}{\Delta t}=-\frac{\nabla p}{\rho}
\end{aligned}
$$

where $S h=U / c$ is the Strouhal number and $p$ is the pressure of the liquid water/gas two-phase system. More detailed information about the model can be found in Inamuro et al. (2004) and Tabe et al. (2009).

\subsection{Calculation process of the Poisson equation}

Previously, the Poisson equation was solved by using the SOR method, owing to its easy implementation (Tabe, 
2009) and faster calculation speed, compared to the LBM scheme. In the SOR method, the calculation that uses the co-location grid (Fig. 1(b)) to solve the Poisson equation induces spatial pressure oscillations. To prevent such oscillations, pressure discretization formulations were derived for the solution of the Poisson and velocity modification equations, based on two types of staggered grids (Fig. 1(c) and 1(d)) that shift the velocity definition points to the boundaries of the conservation volume units.

For Staggered Grid 1, shown in Fig. 1, the pressure at point $(i, j)$ can be estimated based on the following:

$p_{i, \mathrm{j}}^{A}=\frac{\sum_{A} \frac{p}{\rho}-\left(U^{*}{ }_{2}-U^{*}{ }_{4}+U^{*}{ }_{3}-U^{*}{ }_{5}\right)}{\frac{1}{\rho_{2}}+\frac{1}{\rho_{4}}+\frac{1}{\rho_{3}}+\frac{1}{\rho_{5}}}$

For Staggered Grid 2, shown also in Fig. 1, the pressure at point $(i, j)$ is

$p_{i, j}^{B}=\frac{\sum_{B} \frac{p}{\rho}-\sqrt{2}\left(U^{*}{ }_{6}-U_{8}^{*}+U_{9}^{*}-U^{*}\right)}{\frac{1}{\rho_{6}}+\frac{1}{\rho_{7}}+\frac{1}{\rho_{8}}+\frac{1}{\rho_{9}}}$

Here,

$$
\begin{aligned}
& \rho_{2}=\frac{\rho_{i+1, j}+\rho_{i, j}}{2}, \quad \rho_{4}=\frac{\rho_{i-1, j}+\rho_{i, j}}{2}, \quad \rho_{3}=\frac{\rho_{i, j+1}+\rho_{i, j}}{2}, \quad \rho_{5}=\frac{\rho_{i, j-1}+\rho_{i, j}}{2} \\
& U_{2}^{*}=\frac{u_{i, j}^{*}+u_{i+1, j}^{*}}{2}, \quad U_{3}^{*}=\frac{v_{i, j}^{*}+v_{i, j+1}^{*}}{2}, \quad U_{4}^{*}=\frac{u_{i-1, j}^{*}+u_{i, j}^{*}}{2}, \quad U_{5}^{*}=\frac{v_{i, j-1}^{*}+v_{i, j}^{*}}{2} \\
& \rho_{6}=\frac{\rho_{i+1, j+1}+\rho_{i, j}}{2}, \rho_{7}=\frac{\rho_{i-1, j+1}+\rho_{i, j}}{2}, \rho_{8}=\frac{\rho_{i-1, j-1}+\rho_{i, j}}{2}, \rho_{9}=\frac{\rho_{i+1, j-1}+\rho_{i, j}}{2} \\
& U_{6}^{*}=\frac{\frac{u_{i, j}^{*}+v_{i, j}^{*}}{\sqrt{2}}+\frac{u_{i+1, j+1}^{*}+v_{i+1, j+1}^{*}}{\sqrt{2}}}{2}, U_{7}^{*}=\frac{\frac{u_{i, j}^{*}-v_{i, j}^{*}}{\sqrt{2}}+\frac{u_{i-1, j+1}^{*}-v_{i-1, j+1}^{*}}{\sqrt{2}}}{2}, \\
& U_{8}^{*}=\frac{\frac{u_{i, j}^{*}+v_{i, j}^{*}}{\sqrt{2}}+\frac{u_{i-1, j-1}^{*}+v_{i-1, j-1}^{*}}{\sqrt{2}}}{2}, U_{9}^{*}=\frac{\frac{u_{i, j}^{*}-v_{i, j}^{*}}{\sqrt{2}}+\frac{u_{i+1, j-1}^{*}-v_{i+1, j-1}^{*}}{\sqrt{2}}}{2} \\
& \sum_{A} \frac{p}{\rho}=\frac{p_{i+1, j}}{\rho_{2}}+\frac{p_{i-1, j}}{\rho_{4}}+\frac{p_{i, j+1}}{\rho_{3}}+\frac{p_{i, j-1}}{\rho_{5}} \quad \sum_{B} \frac{p}{\rho}=\frac{p_{i+1, j+1}}{\rho_{6}}+\frac{p_{i-1, j-1}}{\rho_{7}}+\frac{p_{i+1, j-1}}{\rho_{9}}+\frac{p_{i-1, j+1}}{\rho_{8}}
\end{aligned}
$$

The subscripts $i$ and $j$ represent the positions of the lattice points along the $x$ and $y$ directions.

\section{Simulation results}

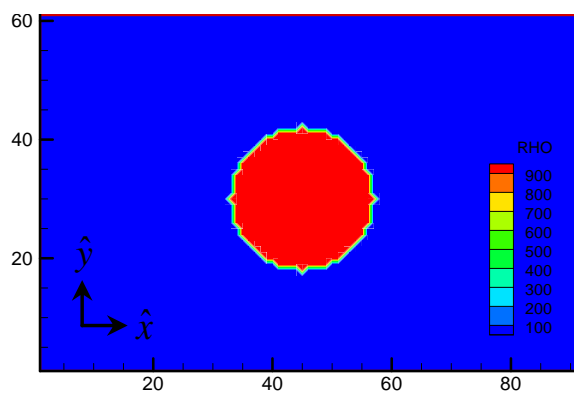

(a) Initial assignment of the two phases

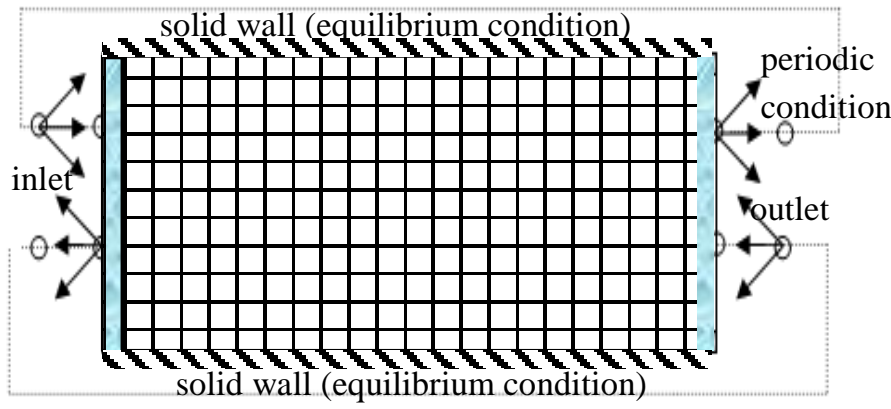

(b) Boundary conditions

Fig. 2 Initial and boundary conditions. 


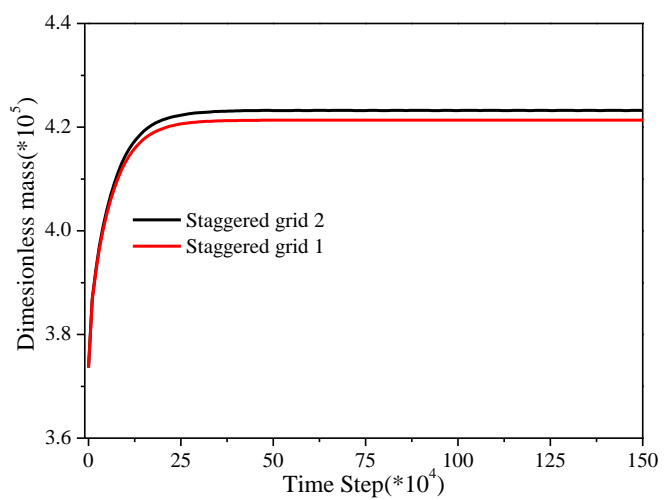

Fig. 3 Mass evolution histories of the water droplet.

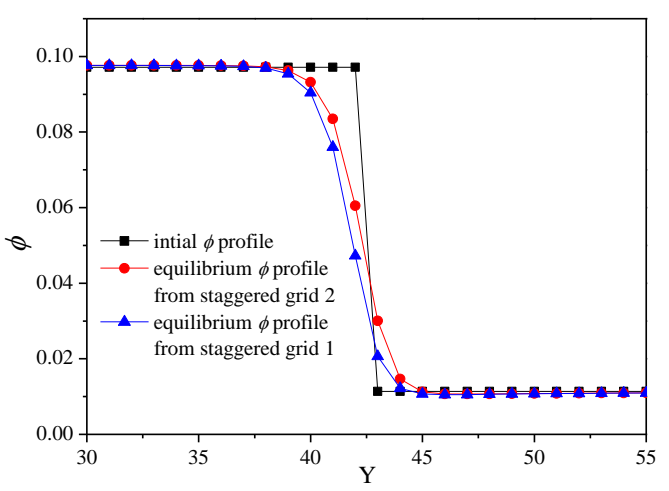

Fig. 4 Interface profiles of the two-phase system.

According to the parameters adopted in Eq. (8), the initial values for the liquid phase and gas phase of the van der Waals fluid are $\phi_{L 0}=9.714 \times 10^{-2}, \phi_{G 0}=1.134 \times 10^{-2}$, respectively. In the simulation, the values $\phi_{L}=9.20 \times 10^{-2}$ and $\phi_{G}$ $=1.15 \times 10^{-2}$ are used to distinguish the liquid water and gas phases. The densities of liquid water and gas are $\rho_{L}=$ $996.62 \mathrm{~kg} / \mathrm{m}^{3}, \quad \rho_{G}=1.1763 \mathrm{~kg} / \mathrm{m}^{3}$, whereas the corresponding viscosities are $\mu_{L}=8.544 \times 10^{-4} \mathrm{~Pa} \cdot \mathrm{s}$ and $\mu_{G}=$ $1.862 \times 10^{-5} \mathrm{~Pa} \cdot \mathrm{s}$, respectively. Moreover, the interface tension is $\sigma=0.07288 \mathrm{~N} \cdot \mathrm{m}, \kappa_{f}=0.5, \kappa_{g}=4.916 \times 10^{-8}$ and the characteristic particle speed $c$ is $200 \mathrm{~m} / \mathrm{s}$. A square lattice $\left(d x=d y=1.33 \times 10^{-4} \mathrm{~m}\right)$ was used in the LBM evolution. A water droplet with a radius of $R=12 d x$ was placed in the center of the calculation domain, as shown in Fig. 2(a). In this figure, the red region is the liquid water and the blue region is the corresponding gas region. The left and right sides of the domain are set as a periodic boundary condition, whereas the top and bottom sides of the domain are set as solid walls, as shown in Fig. 2(b). The lengths of the calculation domain along the $\mathrm{x}$ and the $\mathrm{y}$ directions are equal to $91 d x$ and $61 d x$.

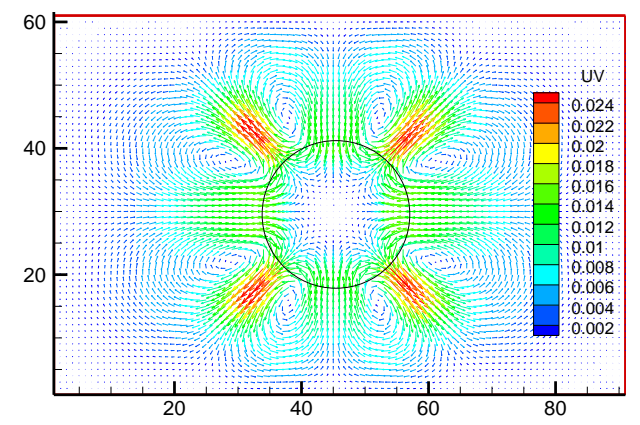

(a) Staggered grid 1

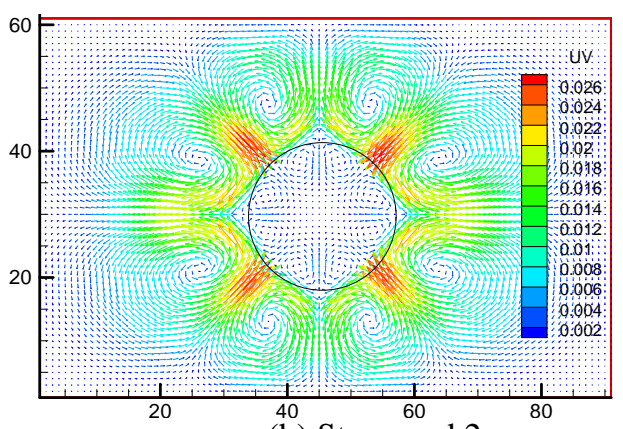

(b) Staggered 2

Fig. 5 Velocity field in steady state.

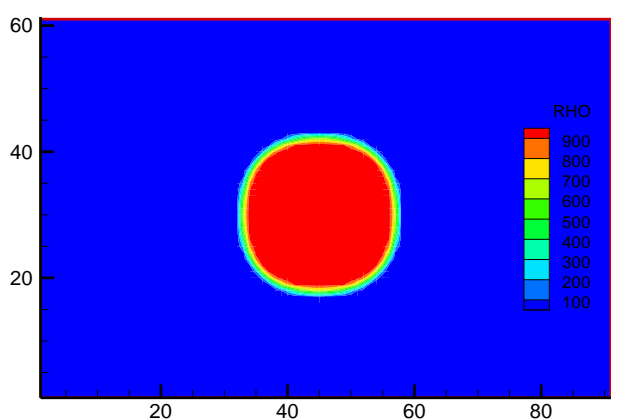

(a) Staggered grid1

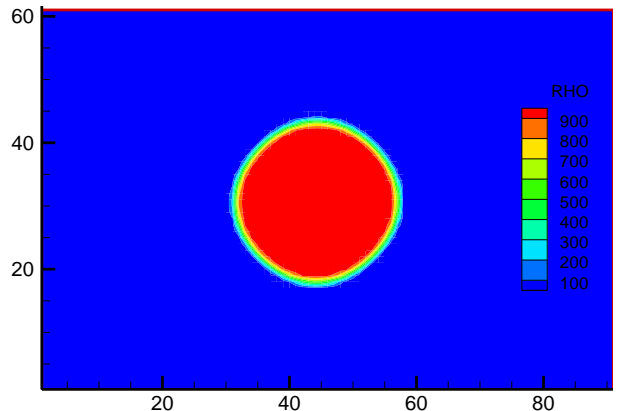

(b) Staggered grid 2

Fig. 6 Density distribution in steady state.

In the simulation, an unsteady stage was observed before the simulation reached the steady state. During this process, the mass of the water droplet increased rapidly, as illustrated in Fig. 3, and an appropriate interface between the liquid and the gas phases with a smooth density distribution inside it was formed, as shown in Fig. 4. For each type of the staggered grids, there is a specific type of a spurious velocity field, as shown in Fig. 5. In this figure, the black 
circle represents the iso-surface where $\rho=\left(\rho_{G}+\rho_{L}\right) / 2$, or $\phi=\left(\phi_{G}+\phi_{L}\right) / 2$. Moreover, shape deformation occurred in the unsteady stage and remained in the steady stage, as shown in Fig. 6.

\section{Discussion}

The equilibrium distribution function of LBM for the ideal gas, which does not take the intermolecular interactions into consideration, based on the $2 \mathrm{D}$ square lattice, can be written in the form of Eq. (22). The variable $\lambda$ used in this section is a free parameter used to represent $\phi$ and $\rho$.

$$
f_{i}^{e q}=\lambda E_{i}\left[1+3 c_{i \alpha} \cdot \boldsymbol{u}+\frac{9}{2}\left(c_{i \alpha} \cdot \boldsymbol{u}\right)^{2}-\frac{3}{2} u^{2}\right]
$$

In the two-phase simulation, the intermolecular interaction of the non-ideal gas must be taken into consideration. According to the Cahn-Hilliard free-energy theory for one-component two-phase fluid, the intermolecular force term in the momentum equation, or the LBM distribution function used to calculate the velocity, can be expressed as follows:

$$
F_{\alpha}=\frac{\partial}{\partial x_{\alpha}}\left(p_{0}-\kappa \lambda \frac{\partial^{2} \lambda}{\partial x_{\alpha}^{2}}-\frac{\kappa}{2}\left(\frac{\partial \lambda}{\partial x_{\alpha}}\right)^{2}\right) \delta_{\alpha \beta}+\kappa \frac{\partial}{\partial x_{\alpha}}\left(\frac{\partial \lambda}{\partial x_{\alpha}} \frac{\partial \lambda}{\partial x_{\beta}}\right)
$$

where, $p_{0}$ is the thermodynamic pressure and $\kappa$ is the interfacial tension related constant. In this paper, a van der Waals fluid is described by $\phi, \kappa=\kappa_{f}$ and $p_{0}=p_{0}(\phi)$, whereas for the liquid water/gas two-phase system, $\kappa=\kappa_{g}$ and $p_{0}=p_{0}(\rho)$. Because of the different definitions and arrangements between the pressure and the interfacial tension parts in Eq. (23), one stress form of the intermolecular force can be written as

$$
\begin{gathered}
F_{\alpha}^{S 1}=\frac{\partial}{\partial x_{\alpha}}\left(p_{0}-\kappa \lambda \frac{\partial^{2} \lambda}{\partial x_{\alpha}^{2}}\right) \delta_{\alpha \beta}-\kappa \frac{\partial}{\partial x_{\alpha}}\left(\frac{1}{2}\left(\frac{\partial \lambda}{\partial x_{\alpha}}\right)^{2} \delta_{\alpha \beta}-\frac{\partial \lambda}{\partial x_{\alpha}} \frac{\partial \lambda}{\partial x_{\beta}}\right) \\
f_{i}^{e q}=H_{i} \phi+F_{i}\left[p_{0}-\kappa_{f} \phi \frac{\partial^{2} \phi}{\partial x_{\alpha}^{2}}-\frac{\kappa_{f}}{6}\left(\frac{\partial \phi}{\partial x_{\alpha}}\right)^{2}\right]+3 E_{i} \phi c_{i \alpha} u_{\alpha}+E_{i} \kappa_{f} G_{\alpha \beta}(\phi) c_{i \alpha} c_{i \beta}
\end{gathered}
$$

The superscript $S 1$ in the term $F_{\alpha}^{S 1}$ represents the first stress form, while the first and the second terms on the right hand side of Eq. (24) represent the corresponding forms of the pressure gradient force and the interfacial tension force, respectively. This stress form of the intermolecular force is adopted by Swift et al. (1995) and Inamuro et al. (2000), and is added to Eq. (22) to represent the non-ideal gas effect. In Inamuro's model for large density ratios (Inamuro, et al., 2004; Tabe, et al., 2009), such form is also adopted for the distribution function $f_{i}$ to get the order parameter $\phi$, but an additional term was added to the pressure part of Eq. (24) to reduce the mobility. Moreover, the terms in Eq. (22) that are not related to mass conservation are removed. Equivalently, Eq. (5) is comprised of the terms from the Cahn-Hilliard equation and an advection term. Such treatments make Eq. (5) a type of a level-set method funcion. From this equation it can also be found that the distribution for $\phi$ (or $\rho$ ), or the droplet shape, is determined by the pressure gradient, the interface tension force and the velocity effect.

As pointed out by Yang and Fleming (1976), Lee and Lin (2005), the $p_{H}$ works as the pressure in the interface and it was defined as the hydrodynamic pressure:

$$
p_{H}=p_{0}-\kappa \rho \frac{\partial^{2} \lambda}{\partial x_{\alpha}^{2}}+\frac{\kappa}{2}\left(\frac{\partial \lambda}{\partial x_{\alpha}}\right)^{2}
$$

The intermolecular force described by Eq. (23) can be rewritten into the form of Eq. (26), namely:

$$
\begin{gathered}
F_{\alpha}^{S 2}=\frac{\partial}{\partial x_{\alpha}}\left(p_{0}-\kappa \lambda \frac{\partial^{2} \lambda}{\partial x_{\alpha}^{2}}+\frac{\kappa}{2}\left(\frac{\partial \lambda}{\partial x_{\alpha}}\right)^{2}\right) \delta_{\alpha \beta}-\kappa \frac{\partial}{\partial x_{\alpha}}\left(\frac{1}{2}\left(\frac{\partial \lambda}{\partial x_{\alpha}}\right)^{2}\right) \delta_{\alpha \beta}-\kappa \frac{\partial}{\partial x_{\alpha}}\left(\frac{1}{2}\left(\frac{\partial \lambda}{\partial x_{\alpha}}\right)^{2} \delta_{\alpha \beta}-\frac{\partial \lambda}{\partial x_{\alpha}} \frac{\partial \lambda}{\partial x_{\beta}}\right) \\
g_{i}^{e q}=E_{i}\left[1+3 c_{i \alpha} u_{\alpha}-\frac{3}{2} u_{\alpha} u_{\alpha}+\frac{9}{2} c_{i \alpha} c_{i \beta} u_{\alpha} u_{\beta}+\frac{3}{2}\left(\tau g-\frac{1}{2}\right) \times \Delta x\left(\frac{\partial u_{\beta}}{\partial x_{\alpha}}+\frac{\partial u_{\alpha}}{\partial x_{\beta}}\right) c_{i \alpha} c_{j}\right] \\
-\frac{1}{2} F_{i} \frac{\kappa_{g}}{\rho}|\nabla \rho|^{2}+E_{i} \frac{\kappa_{g}}{\hat{\rho}} G_{\alpha \beta}(\rho) c_{i \alpha} c_{i \beta}
\end{gathered}
$$


where the superscript $S 2$ in the $F_{\alpha}^{S 2}$ term represents the second stress form of the intermolecular force, a form that was adopted by Inamuro et al. (2004) to extract the predicted velocity. As shown above, the intermolecular force terms of the non-ideal gas were introduced into Eq. (22) with some special treatments. The $\tau_{g}$ related term in Eq. (6) represents the viscosity related force term, and the hydrodynamic pressure part of the intermolecular force was not included. The reason is that, according to the asymptotic analysis, at low Mach numbers the pressure of the two-phase flow can be split into two parts, namely, the thermodynamic pressure that is uniform in space, and the hydrodynamic pressure that is several orders of magnitude smaller than the thermodynamic pressure (Lee and Lin, 2005). Further, the calculation of the $p_{H}$ in the intermolecular force term increases the numerical instability. In the small density ratio case, the hydrodynamic pressure $p_{H}$ term can be usually omitted. However, in the large density ratio case, the $p_{H}$ term becomes much larger, and its effect cannot be neglected, otherwise, the continuity equation cannot be satisfied in the interface region. Instead of calculating the hydrodynamic pressure defined by Eq. (25) and adding it to Eq. (6), the fractional step method based on the Poisson equation (Eqs. (11) and (12)) is used to extract the $p_{H}$ value and improve the predicted velocity. Therefore, in this analysis, the pressure in the Poisson equation is the hydrodynamic pressure.

\subsection{Origins of spurious velocity}

From the mathematical standpoint, the treatments of the intermolecular force terms in Eqs. (23), (24), and (25) are the same. Therefore, the calculation of these equations can be divided into two parts: calculation of (1) the gradient of scalar(s) (2) the gradient of the non-isotropic tensor. Both Wagner (2003) and Lee and Fischer (2006) proved that the truncation error between the two parts is the origin of the spurious velocity in the free-energy-based LBM model, and it was defined as the incompatible discretization error. Additionally, the anisotropic discretization of the derivatives in the intermolecular force terms is also a source of spurious velocity. It has been found that the magnitude of the spurious velocity can be significantly decreased by computing the derivatives with higher isotropic discretization schemes in the free-energy-based LBM model (Seta and Okui, 2007; Pooley and Furtado, 2008).

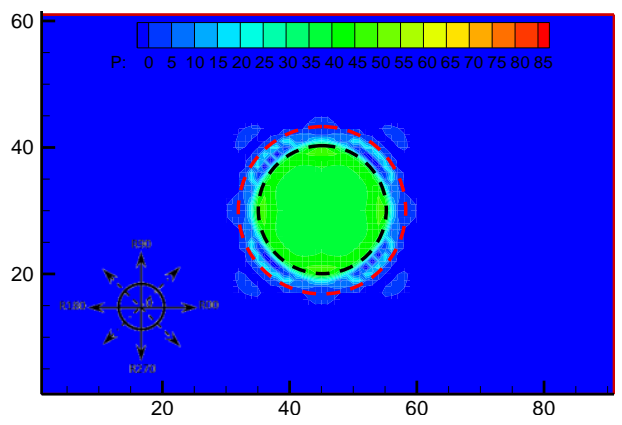

(a) Staggered grid 1

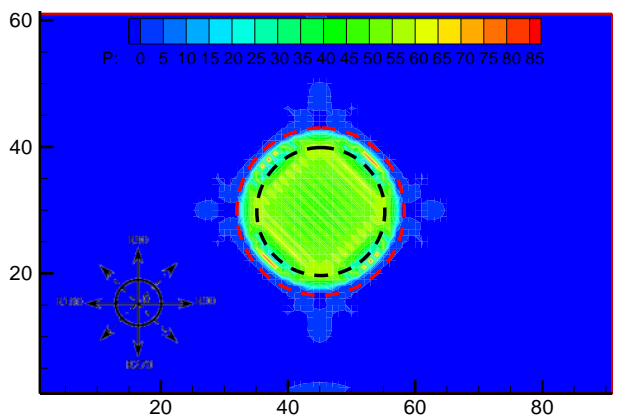

(b) Staggered grid 2

Fig. 7 Hydrodynamic pressure field calculated from Eq. (15) and (16).

The calculation of the hydrodynamic pressure from the Poisson equation by the SOR method with the staggered grids is a new source of spurious velocity. It belongs to the category of anisotropic discretization error. As shown in Fig. 5, when different staggered grids were used in the calculation, different type of spurious velocity fields were observed. The staggered grid 1 that uses the predicted velocity in the orthogonal directions for estimation of the $p_{H}$ created an orthogonal type of spurious velocity field. The staggered grid 2 that uses the predicted velocity in the diagonal directions created a diagonal type of spurious velocity field. If the derivatives in the hydrodynamic pressure (Eq. (25)) are calculated through an isotropic discretization scheme, the $p_{H}$ value should distribute inside and outside the droplet isotropically, as the thermodynamic pressure $p_{0}$ in the equation defining $p_{H}$ can be calculated without any error by the equation of state (EOS, Eq. (8)). Figure 7 shows the hydrodynamic pressure field calculated from Eq. (15) and (16), based on the iterative SOR method. In Fig. 7, the dotted black line is the iso-surface of $\phi=\phi_{L}$ or $\rho=\rho_{L}$, the dotted red line is the iso-surface of $\phi=\phi_{G}$ or $\rho=\rho_{G}$, and the region between them is the interface region. Figure 8 shows the corresponding pressure distributions across the center of the simulated droplet along $0^{\circ}$ and $45^{\circ}$ directions, as shown in the orientation of Fig. 7. In this figure, the dotted yellow line is the expected pressure inside the water droplet which is predicted from the Laplace equation. From these figures, it can be found that in both cases, in the 
interface region, the distribution of $p_{H}$ is anisotropic and the value of $p_{H}$ deviates from the theoretical prediction. Such anisotropic distributions are considered to be the source of the spurious velocity around the interface region of the water droplet. Besides, the $p_{H}$ predicted by using the staggered grid 2, distributed in a chalkboard-type in the liquid region, is believed to be the source of small spurious velocity eddies inside the water droplet.

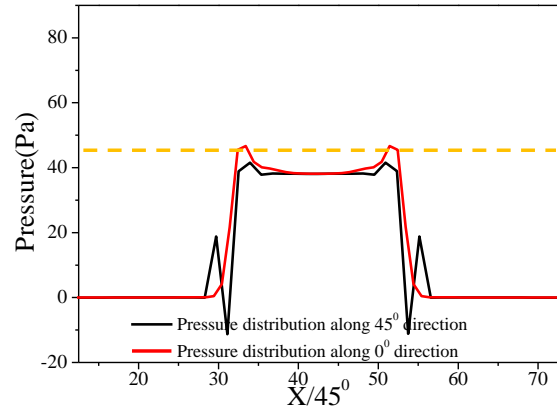

(a) Staggered grid 1

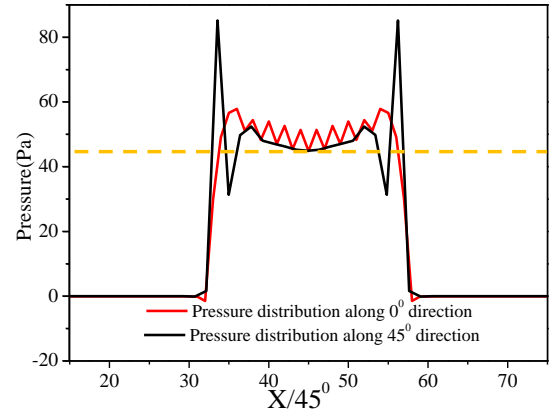

(b) Staggered grid 2

Fig. 8 Hydrodynamic pressure distribution along the $0^{\circ}$ and $45^{\circ}$ directions.

\subsection{Optimazation of the blending factor}
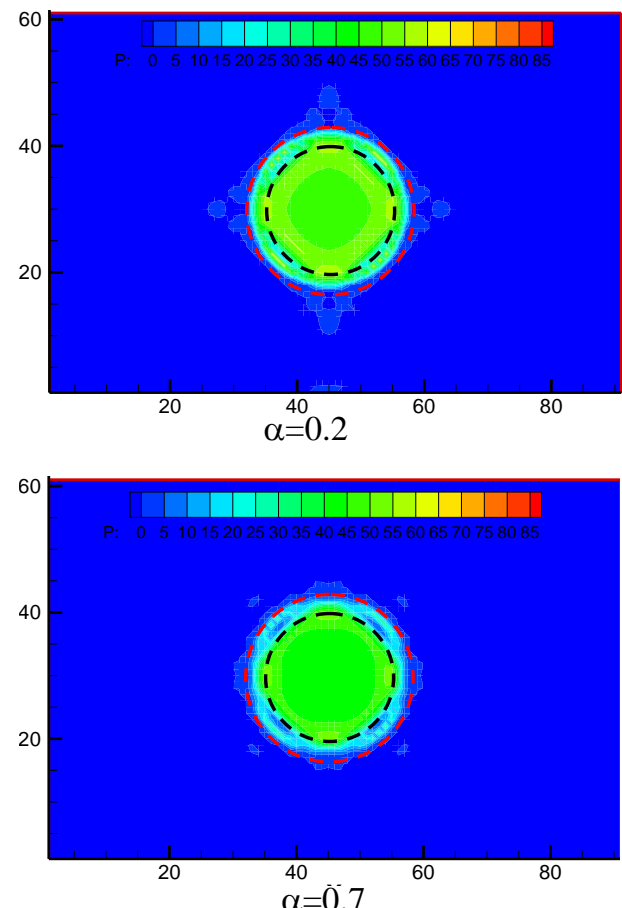
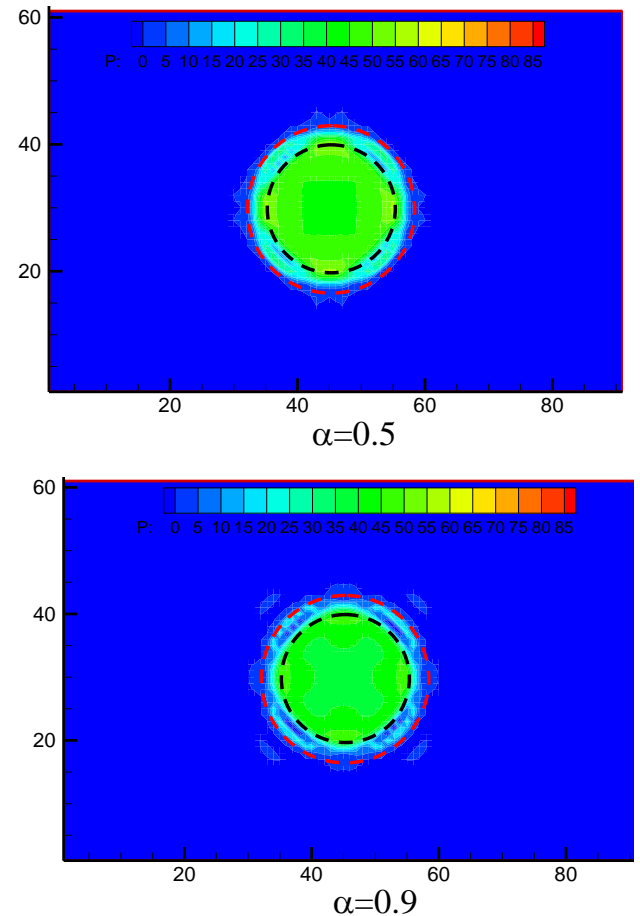

Fig. 9 Hydrodynamic pressure field.

Originally, the hydrodynamic pressure, $p_{H}$, was solved from the Poisson equation by Inamuro et al. (2004). This was accomplished in the framework of the LBM scheme with a co-located grid that defined the pressure and the velocity within the same grid point, as also shown in Fig. 1(b). In this case, all the velocity components along all directions (including the diagonal and orthogonal directions) were taken into account. Such treatment makes the predicted $p_{H}$ to be associated with a better isotropy and accuracy than the corresponding estimates calculated with the staggered grids, discussed earlier.

Adopting the staggered grid 1 in the discretization equation of the Poisson equation for the iterative SOR method means that only the velocity components along the orthogonal directions were taken into account. Correspondingly, adopting the staggered grid 2, means that only the velocity components along the diagonal direction were considered. In order to increase the isotropy in the calculation process of the hydrodynamic pressure, a possible way is to combine 
the velocity components from the two types of staggered grids together. The problem that arises, however, relates to the determination of the ratio for each type of the staggered grid employed in the discretization equation of the Poisson equation. As for the Poisson equation solved by the LBM scheme where the co-locate grid is used, the weight ratio of velocity components used in the calculation along the diagonal and the orthogonal directions was 1/9:1/36. A primary question that arises is whether the same ratio value would be suitable to be used (or not) in the discretization equation for the SOR method for blending velocity components from the two types of staggered grids.

To test this idea, based on the discretization equations (Eq. (15) and (16)) used in the SOR iteration with the staggered grid 1 and the staggered grid 2, the velocity components from the two types of staggered grids are blended together. The variable $\alpha$ in the following equations is defined as the blending factor:

$\alpha+(1-\alpha)=1$

$\alpha \cdot p_{i, j}^{A}+(1-\alpha) \cdot p_{i, j}^{B}=p_{i, j}$

Combining equations Eq. (15), Eq. (16), with some transformations, Eq. (28) can be rewritten as

$$
p_{i, j}=\frac{\alpha\left(\sum_{A} \frac{p}{\rho}-\left(U^{*}{ }_{2}-U^{*}{ }_{4}+U^{*}{ }_{3}-U^{*}{ }_{5}\right)\right)+(1-\alpha)\left(\sum_{B} \frac{p}{\rho}-\sqrt{2}\left(U^{*}{ }_{6}-U_{8}^{*}+U_{9}^{*}-U^{*}{ }_{7}\right)\right)}{\alpha\left(\frac{1}{\rho_{2}}+\frac{1}{\rho_{3}}+\frac{1}{\rho_{4}}+\frac{1}{\rho_{5}}\right)+(1-\alpha)\left(\frac{1}{\rho_{6}}+\frac{1}{\rho_{7}}+\frac{1}{\rho_{8}}+\frac{1}{\rho_{9}}\right)}
$$
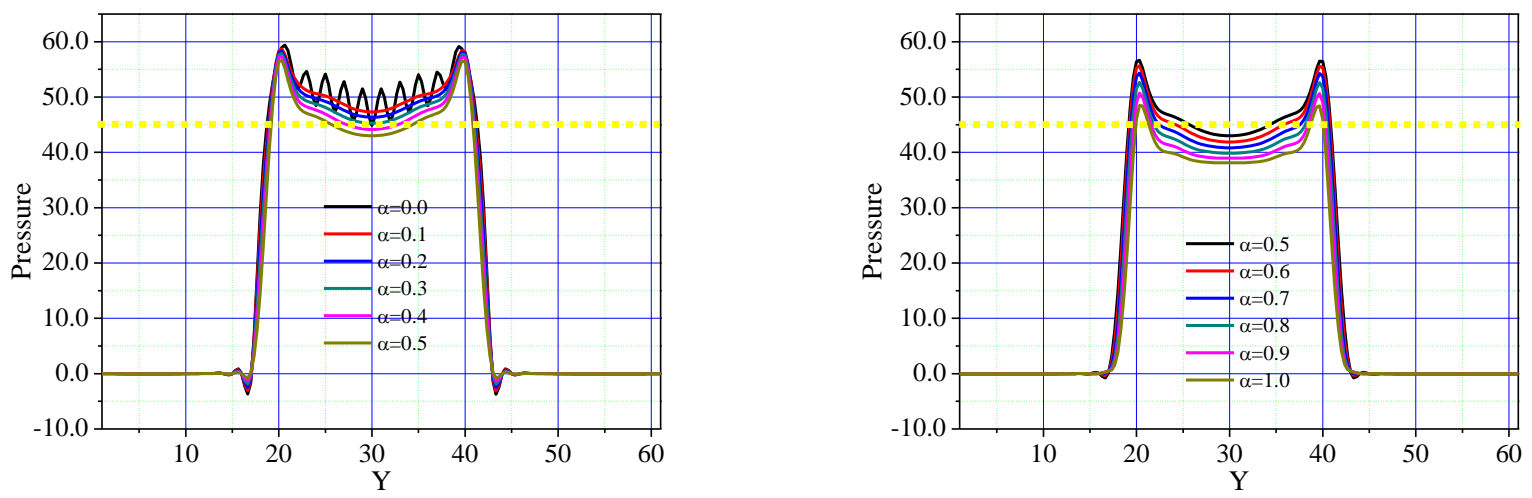

Fig. 10 Hydrodynamic pressure distributions across the center of the calculation domain along the y direction

In order to obtain the appropriate blending factor that can elicit the most isotropic and the most accurate value of the hydrodynamic pressure, eleven $\alpha$ values were tested ranging from 0.0 to 1.0 , with a step interval of 0.1 . The choice of $\alpha=0.0$ corresponds to the case of adopting the staggered grid 2 only in the discretization equation of the Poisson equation, whereas $\alpha=1.0$ corresponds to the case of adopting the staggered grid 1 only. It is found that starting from $\alpha=0.0$ (or $\alpha=1.0$ ) gradually increases the ratio of velocity component in the discretization equation of the Poisson equation from another staggered grid, the predicted hydrodynamic pressure, $p_{H}$, becomes more isotropic and accurate. When $\alpha=0.5$, which means that the velocity components in the discretization equations of the Poisson equation from the two types of staggered grids are equally blended, $p_{H}$ can attain the most isotropic distribution and the most accurate value, although the anisotropy cannot be totally removed at the interface region of the droplet. Figure 9 shows the predicted $p_{H}$ field based on the simulation with a selection of different $\alpha$ values. The results of Fig. 9, in combination with those of Fig. 7, indicate that the isotropy of the $p_{H}$ increases when $\alpha$ approaches 0.5 , irrespective of the starting value (that is, either from 0.0 or from 1.0). Figure 10 shows the predicted hydrodynamic pressure distribution across the center of the calculation domain, along the y direction. From this figure, it is seen that as a value of $\alpha$ becomes close to around 0.0 , the solution tends to predict a higher averaged $p_{H}$ value inside the water droplet, whereas a corresponding value of $\alpha$ around 1.0 tends to predict a smaller value. The averaged $p_{H}$ value inside the droplet can be accurately predicted when $\alpha=0.5$. Corresponding to Fig. 9, Fig. 11 shows the spurious velocity field predicted by different blending factors. In combination with Fig. 5, it can be found that starting from $\alpha=0.0$ (or $\alpha=1.0$ ), the magnitude of the spurious velocity gradually decreases with the increase of contribution from another staggered 
grid. The predicted hydrodynamic pressure with the smallest anisotropy makes the magnitude of the spurious velocity field the smallest. When $\alpha=0.5$, where the contribution from the two types of staggered grids are blended equally, the magnitude of the predicted spurious velocity is decreased to half of its original value.
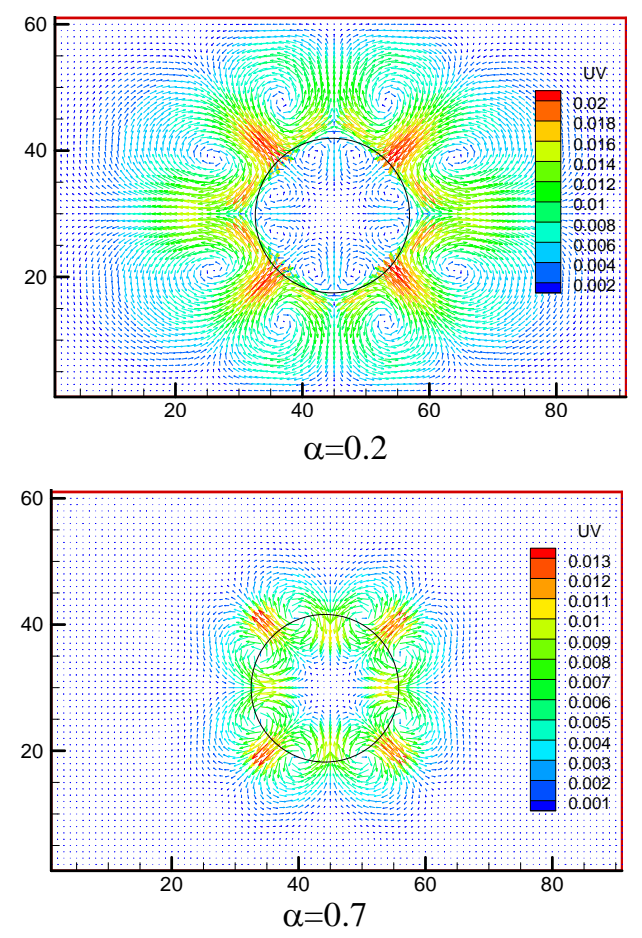
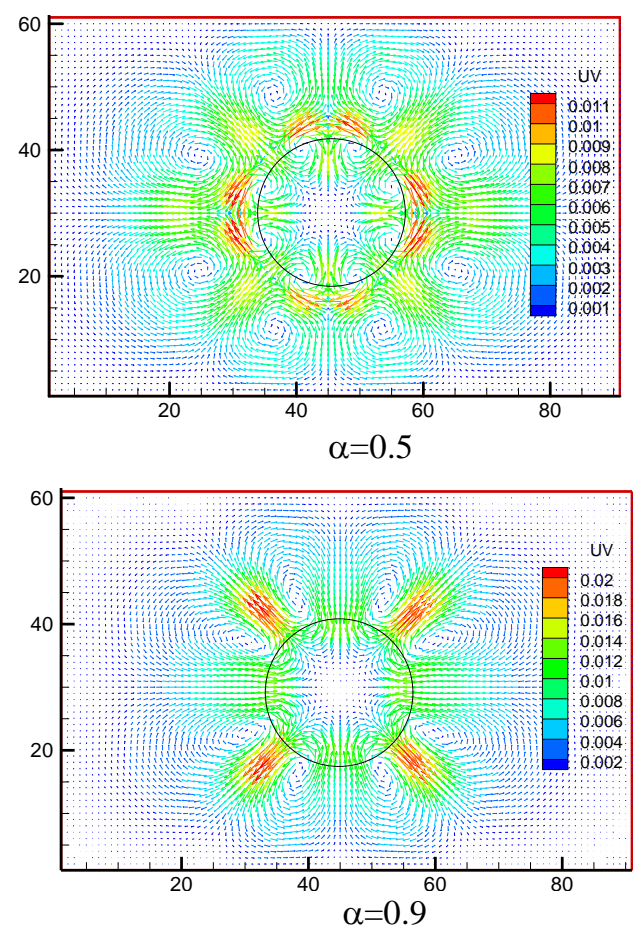

Fig. 11 Spurious velocity fields with a typical blending factor $\alpha$.

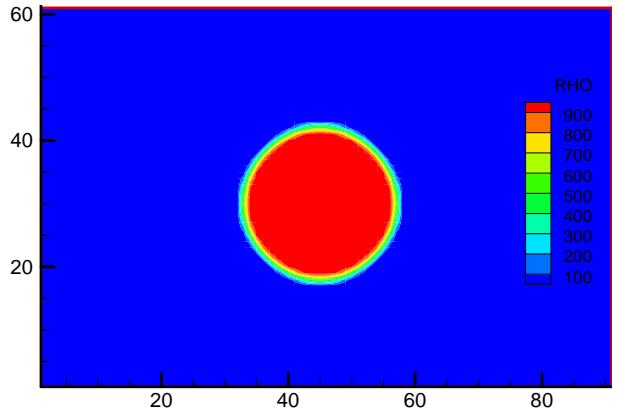

(a) $\alpha=0.2$

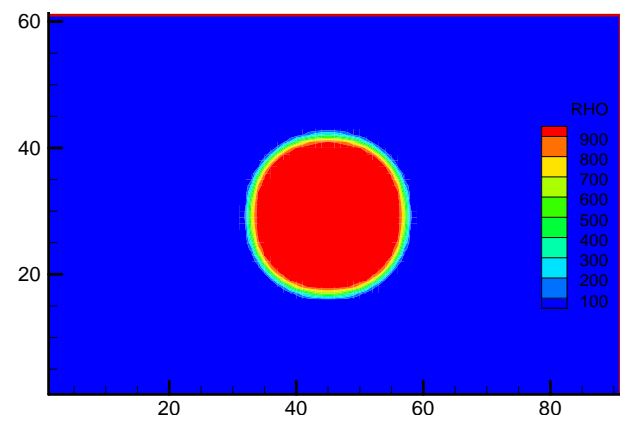

(c) $\alpha=0.7$

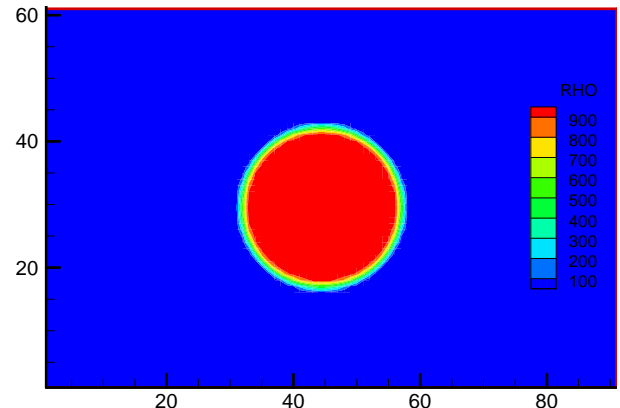

(b) $\alpha=0.5$

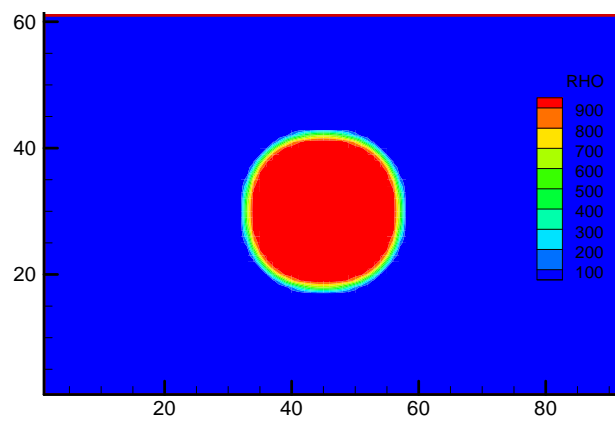

(d) $\alpha=0.9$

Fig. 12 Density distribution with a typical blending factor $\alpha$.

As illustrated in Figs. 5 and 6, the existence of the spurious velocity field leads to the deformation of the simulated water droplet. When the staggered grid $1(\alpha=1.0)$ was used in the simulation, it created a spurious velocity field where the velocity vectors flow inward along orthogonal directions and outward along the diagonal directions, in the vicinity 
of the liquid/gas interface. Meanwhile, the shape of the droplet deforms along the direction of the velocity vectors. When the staggered grid $2(\alpha=0.0)$ was used, the velocity vectors flow in the opposite directions and the shape of droplet deforms correspondingly. Figure 12 shows different shapes of the simulated water droplets in the steady state with a typical blending factor value, which corresponds to the $\alpha$ value used in Fig. 11. From this figure, it can be seen that, with changes in the structures (spurious velocity vector directions and positions) and the magnitude of the spurious velocity field, the droplet deforms simultaneously. Therefore, the advection effect caused by the spurious velocity makes shape deformation. The deformation of the droplet is categorized into two types: (1) as $\alpha$ approaches to 0.0 it leads to a diamond-like shape, and (2) as $\alpha$ becomes near 1.0 it leads to a rectangular shape. Therefore, the radius length difference along eight directions is used to measure the degree of shape deformation, as shown by the insert of Fig. 13(a). In this figure, the yellow dotted lines represent the initial radial length, while R2-R9 represent the different radial directions. From this figure, it is seen that an $\alpha$ value of around 0.5 yields the minimum shape deformation, where an $\alpha$ value approaching to 1.0 leads to a larger deformation than the case where $\alpha$ is near 0.0 . The $\alpha$ values near 0.0 and 1.0 are usually associated with the same magnitude of spurious velocity, as illustrated in Figs. 5 and 11 . The difference in the degree of shape deformation comes from the hydrodynamic pressure field inside the droplet. The case of staggered grid $2(\alpha=0.0)$ usually leads to a chalkboard-like pressure inside the droplet. Such a spatial oscillation would induce small spurious velocity eddies inside the droplet flowing in the opposite directions to those of the main spurious velocity eddies around the interface region. As shown in Fig. 11, in all the blending cases that the velocity component in the discretization equation of the Poisson equation from the staggered grid 2 dominates, such small eddies always exist. These opposite velocity vectors inside the droplet decrease the driving effect of the main spurious velocity eddies, thus resulting in a droplet possessing a smaller degree of shape deformation, compared to the cases where velocity component from the staggered grid 1 dominates.
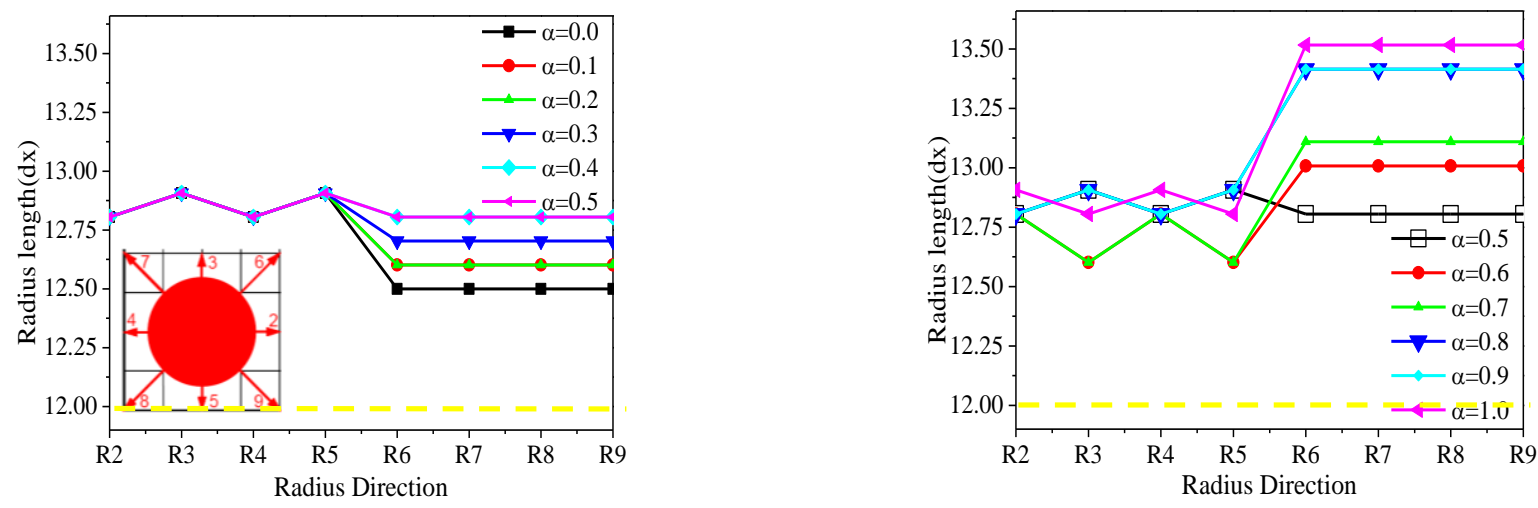

Fig. 13 Radial length distributions of the droplet with different blend factor $\alpha$.

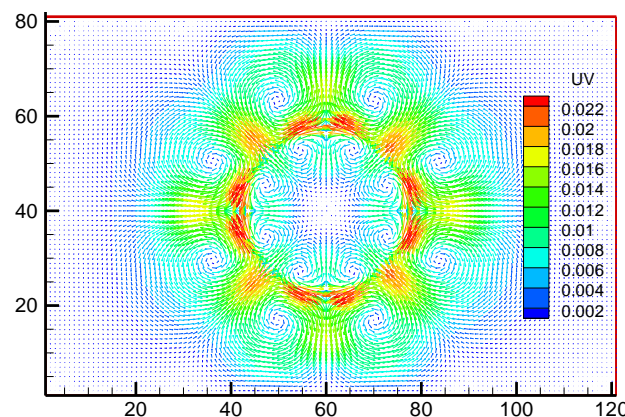

(a) $\alpha=0.5$

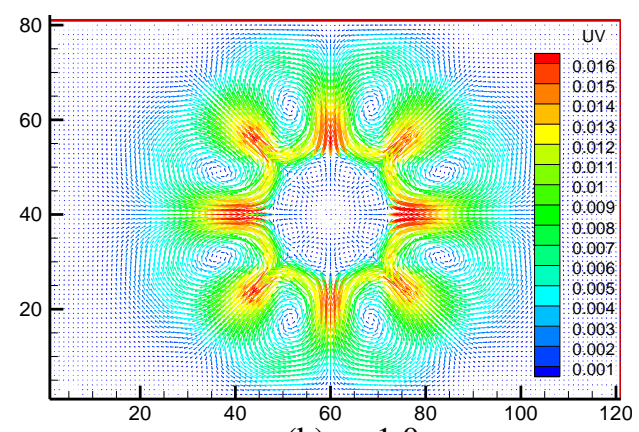

(b) $\alpha=1.0$

Fig. 14 Examples of spurious velocity filed when radius is $16 d x$

Actually, due to the special treatments in this free energy based ratio two-phase flow LBM model for large density ratio, many other factors affects the spurious velocity field and the shape deformation; thus the appropriate blending factor $\alpha$ which can get the smallest spurious velocity and the smallest deformation is no longer to be 0.5 when the dimensionless radius of the droplet changes. Fig. 14 shows spurious velocity field when the radius is $16 d x$ with $\alpha=0.5$ 
and 1.0, in this figures the $\alpha=0.5$ case predicted larger magnitude of spurious velocity than the $\alpha=1.0$. This is because when the range of radius changes, other factors exert different effects on the spurious velocity. In order to avoid these effects and concentrate on the problems related to the calculation of hydrodynamic pressure, this paper only discuss the results of droplet with radius of $12 d x$. The detailed mechanism of the spurious velocity variation with the radius length will be discussed in another paper.

\section{Conclusions}

1. The pressure calculated from the Poisson equation works as the hydrodynamic pressure in the free-energy-based LBM scheme.

2. The hydrodynamic pressure calculated from the discretization equation of the Poisson equation with staggered grids in the iterative SOR method is associated with an anisotropic discretization error, and such type of error is a source of spurious velocity.

3. The anisotropic error can be reduced by blending the velocity components in the discretization equations of the Poisson equation with the two types of staggered grids that make use of the velocity components in the orthogonal and the diagonal directions repectively.

4. The advection effect caused by the spurious velocity leads to droplet deformations.

5. A blending factor $\alpha$ of around 0.5 results in a predicted hydrodynamic pressure with the highest isotropy and accuracy. In this case, the magnitude of the spurious velocity is the smallest and it is about half of the values predicted when $\alpha=0.0$ or 1.0 .

\section{References}

Chiappoini, D., Bella, G., Succi, S., Toschi, F. and Ubertini, S., Improved lattice Boltzmann without parasitic currents for Rayleigh-Taylor instability, Vol.7, No.3 (2010), pp.423-444.

Connington, K. and Lee, T., A review of spurious currents in the lattice Boltzmann method for multiphase flows, Journal of Mechanical Science and Technology, Vol.26, No.12 (2012), pp.3857-3863.

Gong, J., Oshima, N., Kurihara, E. and Tabe, Y., Decision of the best blend proportional factor value of two staggered grids used in LBM for PEFC simulation, Proceeding of the 9th International Conference on Nanochannels, Microchannels, and Minichannels, ASME (2011), pp.239-248.

Gong, J., Oshima, N. and Tabe, Y., The appropriate blend proportional factor value of two staggered grids used in the free energy based LBM With large density ratio, Proceeding of the 11th International Conference on Nanochannels, Microchannels, and Minichannels, ASME (2013), pp. V001T012A006.

Guo, Z.L., Zheng, C.G. and Shi, B.C., Force imbalance in lattice Boltzmann equation for two-phase flows, Physical Review E, Vol.83, No.3 (2011), pp.1-8 (036707).

He, X., Chen, S. and Zhang, R., A lattice Boltzmann Scheme for incompressible multiphase flow and its application in simulation of Rayleigh-Taylor instability, Journal of Computational Physics, Vol.152, No.2 (1999), pp.642-663.

Huang, H., Wang, L. and Lu, X., Evaluation of three lattice Boltzmann models for multiphase flows in porous media, Computers and Mathematics with Applications, Vol.61, No.12 (2011), pp.3606-3617.

Inamuro, T., Konishi, N. and Ogino, F., A Galilean invariant model of the lattice Boltzmann method for multiphase fluid flows using free-energy approach, Computer Physics Communications, Vol.129, No.1-3 (2000), pp.32-45.

Inamuro, T., Ogata, T., Tajima, S. and Konishi, N., A lattice Boltzmann method for incompressible two-phase flows with large density differences, Journal of Computational Physics, Vol.198, No.2 (2004), pp.628-644.

Lee, T. and Lin, C., Astable discretization of the lattice Boltzmann equation for simulation of incompressible two-phase flows at high density ratio, Journal of Computational Physics, Vol.206, No.1 (2005), pp.16-47.

Lee, T. and Fischer, P. F., Eliminating parasitic currents in the lattice Boltzmann equation method for nonideal gases, Physical Review E, Vol.74, No.4 (2006), pp.1-7 (046709).

Moriyama, K. and Inamuro, T., Lattice Boltzmann simulations of water transport from the gas diffusion layer to the gas channel in PEFC, Communications in Computational Physics, Vol.9, (2011), pp.1206-1218.

Pooley, C. M. and Furtado, K., Eliminating spurious velocities in the free energy lattice Boltzmann method, Physical Review E, Vol.77, No.4 (2008), pp.1-9 (046702). 
Ryu, S. and Ko, S., A comparative study of lattice Boltzmann and volume of fluid method for two-dimensional multiphase flows, Nuclear Engineering and Technology, Vol.44, No.6 (2011), pp.623-638.

Salah, Y.B., Tabe, Y. and Chikahisa, T., Two phase flow simulation in a channel of a polymer electrolyte membrane fuel cell using the lattice Boltzmann method, Jounal of Power Sources, Vol.199, No.0 (2012), pp.85-93.

Scarbolo, L., Molin, D., Perlekar, P., Sbragalia, M., Soldati, A. and Toschi, F., Unified framework for a side-by-side comparison of different multi-component algorithms: Lattice Boltzmann vs. phase field model, Journal of Computational Physics, Vol.234 (2012), pp.263-279.

Seta, T. and Okui, K., Effects of truncation error of derivative approximation for two-phase lattice Boltzmann method, Journal of Fluid Science and Technology, Vol.2, No.1 (2007), pp.139-151.

Succi, S., The Lattice Boltzmann Equation for Fluid Dynamics and Beyond (2001), pp.155-176, 179-213, Oxford University Press.

Swift, M.R., Osborn, W.R. and Yeomans, J.M., Lattice Boltzmann simulation of nonideal fluids, Physical Review Letters, Vol.75, No.5 (1995), pp.830-833.

Tabe, Y., Lee, Y., Chikahisa, T. and Kozakai, M., Numerical simulation of liquid water and gas flow in a channel and a simplified gas diffusion layer model of polymer electrolyte membrane fuel cells using the lattice Boltzmann method, Journal of Power Sources, Vol.193, No.1 (2009), pp.24-31.

Wagner, A. J., The origin of spurious velocities in lattice Boltzmann, International Journal of Modern Physics B, Vol.17, No.1-2 (2003), pp.193-196.

Yan, Y. and $\mathrm{Zu}$, Y., A lattice Boltzmann method for incompressible two-phase flows on partial wetting surface with large density ratio, Journal of Computational Physics, Vol.227, No.1 (2007), pp.763-775.

Yang, A.J., Fleming, P.D. and Gibbs, J.H., Molecular theory of surface tension, The Journal of Chemical Physics, Vol.64, No.9 (1976), pp.3732-3747.

Zheng, H.W., Shu, C. and Chew, Y.T., A lattice Boltzmann model for multiphase flows with large density ratio, Journal of Computational Physics, Vol.218, No.1 (2006), pp.353-371. 